Intensivmed 2010 - 47:336-337 DOI 10.1007/s00390-010-0188-z

Online publiziert: 12. Juni 2010

(c) Springer-Verlag 2010

G. Marx

Klinik für Operative Intensivmedizin, Universitätsklinikum der RWTH Aachen

\title{
Hämodynamisches Monitoring
}

tienten durchaus adäquat sein, da dieser Patient noch analgosediert ist. Ebenso kann ein normales HZV abnormal niedrig sein, wenn durch eine bestehende Sepsis ein inflammationsinduzierter Anstieg zur Deckung des Sauerstoffbedarfs notwendig ist.

Substanzen ist die Notwendigkeit einer HZV-Bestimmung allgemein anerkannt, um die Aufrechterhaltung eines ausreichenden intravasalen Volumens und adäquaten Sauerstoffangebots sicherzustellen und konsekutiv Gewebeoxygenierung, Gewebeperfusion und Organfunktion insgesamt zu erreichen. Neben der Beurteilung und Steuerung von kardialer Vorlast sind die wichtigen Messparameter für globale Perfusion flussbasierte Parameter: Sauerstoffangebot und Sauerstoffverbrauch, die Messung des $\mathrm{HZV}$, der zentralvenösen $\left(\mathrm{S}_{\mathrm{zV}} \mathrm{O}_{2}\right)$ Sauerstoffsättigung oder der gemischtvenösen Sauerstoffsättigung $\left(\mathrm{S}_{\mathrm{v}} \mathrm{O}_{2}\right)$. Dagegen besteht keine Korrelation zwischen mittlerem arteriellem Blutdruck und/oder der Herzfrequenz zum Sauerstoffangebot des Patienten.

Klinisch weite Verbreitung hat die Messung des HZV erst durch die Einführung des pulmonalarteriellen Katheters (PAK) durch Swan und Ganz 1970 gefunden. Seit Mitte der 1990er-Jahre wird über das Risiko-Nutzen-Verhältnis des PAK anhaltend diskutiert [1]. Seit dieser Zeit sind einige minimal- oder weniger invasive hämodynamische Monitoringverfahren entwickelt und klinisch eingeführt worden, um das HZV zu messen. Welches HZV ist adäquat für einen Patienten? Bei der Beantwortung dieser Frage muss v. a. bei Intensivpatienten beachtet werden, dass das HZV ein adaptiver Wert ist. So kann ein abnormal niedriger $\mathrm{HZV}$-Wert für einen postoperativen $\mathrm{Pa}$ -

\section{(?) Hämodynamische Monitoringverfahren bieten die Möglichkeit der kontinuierlichen Überwachung verschiedener Parameter}

Ein wichtiger Aspekt der verschiedenen hämodynamischen Monitoringverfahren ist die Möglichkeit der kontinuierlichen Überwachung der verschiedenen Parameter, da hierdurch die Gelegenheit besteht, Veränderungen der Hämodynamik sofort zu erkennen und eine sich dadurch ergebende therapeutische Interventionsmöglichkeit umgehend durchzuführen. Im weiteren Verlauf ermöglicht das hämodynamische Monitoring, kontinuierlich den Erfolg dieser Intervention zu evaluieren.

Im Rahmen des Themenschwerpunktes „Hämodynamisches Monitoring“ in dieser Ausgabe der Zeitschrift „Intensivmedizin und Notfallmedizin" haben 4 Experten sich mit diesem und anderen Aspekten intensiv auseinandergesetzt und Ihnen den gegenwärtigen Wissensstand kompetent und kritisch zusammengefasst. In dem Beitrag von Herrn Bloos über den Stellenwert des perioperativen $\mathrm{S}_{\mathrm{zv}} \mathrm{O}_{2}$-Monitorings wird deutlich, dass die Messung der $\mathrm{S}_{\mathrm{zv}} \mathrm{O}_{2}$ nicht nur bei septischen Patienten Outcome-relevant ist, sondern auch intra- und frühpostoperativ bei Hochrisikochirurgiepatienten potenziell sehr nützlich sein kann. Im nächsten Beitrag beschreibt Herr Heringlake sehr ausgewogen und ausführlich den Stellenwert des pulmonalarteriellen Katheters in der Anästhesiologie und operativen Intensivmedizin. $\mathrm{Da}$ in kontrollierten Studien häufig keine Verbesserung des Patienten-Outcomes durch den Einsatz des PAK belegt werden konnte, wird der Einsatz dieses invasiven Monitoringverfahrens in den letzten Jahren sehr kontrovers diskutiert. Andererseits konnte durch die Verwendung des PAK bei herzchirurgischen Patienten in der postoperativen Phase die Morbidität positiv beeinflusst werden [2]. Chittock et al. [3] analysierten in einer retrospektiven Kohortenstudie bei 7310 kritisch kranken Patienten den Zusammenhang zwischen Einsatz des PAKs und Patienten-Outcome. Diese retrospektive Studie zeigte, dass eine Verbesserung des PatientenOutcome bei schwerstkranken Patienten (APACHE II >31) durch den Einsatz des PAKs möglich ist. Andererseits war der Einsatz des PAKs bei den weniger schwer kranken Patienten (APACHE II <18) mit einem erhöhten Krankenhausletalitätsrisiko assoziiert. Dieses Ergebnis zeigte die Notwendigkeit der Erfassung des Krankheitsschweregrades von Patienten in zukünftigen prospektiven kontrollierten Studien über die Verbesserung des $\mathrm{Pa}$ tienten-Outcome durch den Einsatz des PAK.

Herr Rex diskutiert im nächsten Beitrag eingehend Möglichkeiten und Grenzen neuerer minimal- oder weniger invasiver hämodynamischer Monitoringverfahren zur Messung des HZV. Von besonderer Bedeutung ist bei diesen Verfahren die Validität der Messergebnisse. Abschließend erläutert Herr Wiesenack, welcher Patient im Operationssaal vom 
erweiterten hämodynamischen Monitoring profitieren kann. Hierbei wird sehr deutlich, dass Hochrisikopatienten in der Chirurgie von den Möglichkeiten eines erweiterten hämodynamischen Monitoringverfahrens profitieren können. In mehreren Studien konnte gezeigt werden, dass durch Schlagvolumen-gesteuerte perioperative Volumentherapie die Inzidenz von postoperativen Komplikationen und Krankenhausaufenthalt signifikant reduziert werden konnte [4, 5, 6, $7,8,9]$. Das hämodynamische Monitoring ermöglicht eine patientenadaptierte individuelle Therapie, um ausreichend Volumen zu applizieren und damit eine Hypovolämie zu verhindern. Andererseits kann aber auch eine Volumenüberladung vermieden werden, die $\mathrm{zu}$ Gewebeödem und Anastomosenkomplikationen führen kann. Auch die Optimierung des Sauerstoffangebots führte in mehreren Studien zu einer Reduktion der Morbidität und verkürztem Intensivaufenthalt [10]. So konnten Pölönen et al. [2] zeigen, dass bei kardiochirurgischen Patienten postoperativ eine zielgerichtete Therapie mit Volumentherapie und Inotropika vorteilhaft ist. Interessanterweise wird die vorhandene Evidenz, dass hochrisikochirurgische Patienten sowohl intra- als auch postoperativ häufig vom hämodynamischen Monitoring profitieren können, noch nicht umfassend in die tägliche perioperative Praxis implementiert.

Unabhängig von dem verwendeten Monitoringverfahren ist es wichtig, dass die Messung in einem Behandlungsalgorithmus integriert wird. Die Messung eines hämodynamischen Parameters allein ändert nichts am Outcome des $\mathrm{Pa}$ tienten. Vielmehr ermöglicht die Messung dieser Parameter eine sog. standardisierte „Goal-directed-Therapie“, deren Anwendung den entscheidenden Unterschied für den Patienten darstellen kann.

Es ist wichtig, die Grenzen des erweiterten hämodynamischen Monitorings zu erkennen. Auch wenn die Makrozirkulation adäquat ist, kann eine weiterhin bestehende Störung der Mikrozirkulation mit diesem Monitoringverfahren nicht ausgeschlossen werden. Trotzdem stellen die Erfassung des HZV und der zentralvenösen Sauerstoffsättigung wichtige Eckpunkte in der hämodynamischen Evaluierung und Therapie des kritisch kranken Patienten im Operationssaal und auf der Intensivstation dar.

Ich wünsche eine interessante und aufschlussreiche Lektüre.

Ihr

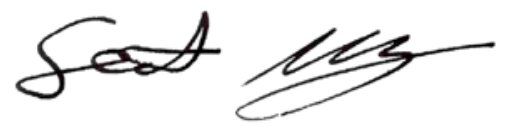

G. Marx

\section{Korrespondenzadresse \\ Prof. Dr. G. Marx}

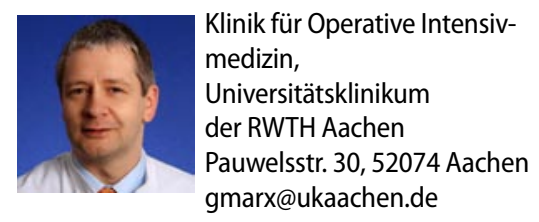

Interessenkonflikt. Der korrespondierende Autor weist auf folgende Beziehungen hin: Edwards Life Sciences und Pulsion Medical Systems haben die Durchführung wissenschaftlicher Projekte von Prof. Dr. G. Marx finanziell unterstützt. Unabhängig hiervon hat Prof. Dr. G. Marx Honorare im Rahmen von Vortragstätigkeiten von diesen Firmen erhalten und ist Berater von Edwards Life Sciences.

\section{Literatur}

1. Ivanov RI, Allen J, Sandham JD, Calvin JE (1997) Pulmonary artery catheterization: a narrative and systematic critique of randomized controlled trials and recommendations for the future. New Horiz 5:268-276

2. Pölönen $P$, Ruokonen $E$, Hippeläinen $M$ et al (2000) A prospective, randomized study of goal-oriented hemodynamic therapy in cardiac surgical patients. Anesth Analg 90:1052-1059

3. Chittock DR, Dhingra VK, Ronco JJ et al (2004) Severity of illness and risk of death associated with pulmonary artery catheter use. Crit Care Med 32:911-915

4. Sinclair S, James S, Singer M (1997) Intraoperative intravascular volume optimization and length of hospital stay after repair of proximal femoral fracture: a randomized controlled trial. BMJ 315:909912

5. Gan TJ, Soppitt A, Maroof M et al (2002) Goal-directed intraoperative fluid administration reduces length of hospital stay after major surgery. Anesthesiology 97:820-826

6. Poeze M, Greve JWM, Ramsay G (2005) Meta-analysis of hemodynamic optimization: relationship to methodological quality. Crit Care 9:R771-779

7. Brienza N, Giglio MT, Marucci M, Fiore T (2009) Does perioperative hemodynamic optimization protect renal function in surgical patients? A metaanalytic study. Crit Care Med 37:2079-2090
8. Lopes MR, Oliveira MA, Pereira VOS et al (2007) Goal-directed fluid management based on pulse pressure variation monitoring during high-risk surgery: a pilot randomized controlled trial. Crit Care 11:R100

9. Mayer J, Boldt J, Mengistu AM et al (2010) Goal-directed intraoperative therapy based on autocalibrated arterial pressure waveform analysis reduces hospital stay in high-risk surgical patients: a randomized, controlled trial. Crit Care 14:R18

10. Pearse R, Dawson D, Fawcett J et al (2005) Early goal-directed therapy after major surgery reduces complications and duration of hospital stay. A randomised, controlled trial [ISRCTN38797445]. Crit Care 9:R687-693 